\title{
Stress condensation in crushed elastic manifolds
}

\author{
Eric M. Krament and Thomas A. Witten \\ The James Franck Institute and the Department of Physics \\ The University of Chicago, Chicago, Illinois 60637
}

(June 29, 2021)

\begin{abstract}
We discuss an $M$-dimensional phantom elastic manifold of linear size $L$ crushed into a small sphere of radius $R \ll L$ in $N$-dimensional space. We investigate the low elastic energy states of 2 -sheets $(M=2)$ and 3 -sheets $(M=3)$ using analytic methods and lattice simulations. When $N \geq 2 M$ the curvature energy is uniformly distributed in the sheet and the strain energy is negligible. But when $N=M+1$ and $M>1$, both energies appear to be condensed into a network of narrow $M-1$ dimensional ridges. The ridges appear straight over distances comparable to the confining radius $R$.
\end{abstract}

03.40.Dz,46.30.Cn,68.60.Bs

It has long been known that a thin elastic plate will develop narrow ridges under a variety of compressive boundary conditions. These ridges may be seen daily in the way a pillowcase or trouser leg deforms to wrap its contents, often exhibiting a diamond pattern familiar from compression studies of thin metal cylinders [1]. The linear scars in a crumpled sheet of paper are also a record of this mechanism [2]. Recently it was discovered that the structure of these ridges could be accounted for using linear elasticity theory, valid in the limit that the ridge length $X$ is much greater than the plate thickness $h$ [3, , 1 . Witten and Li used a scaling argument to predict that the ridge width $w \simeq h^{1 / 3} X^{2 / 3}$ and the total elastic energy $E \simeq Y h^{3}(X / h)^{1 / 3}$ where $Y$ is the Young's modulus [3]. Lobkovsky et al. verified these scaling laws using both numerical simulations and an asymptotic analysis of the Von Karman equations for a thin plate 此. His simulations showed that the material strains and curvatures decay rapidly to zero in the direction transverse to the ridge. The length scale of this decay is the ridge width $w$, which goes to zero with the thickness of the plate. However, these ridges were analyzed only in idealized, symmetrical deformations. Their applicability to stochastically crumpled sheets has not been explicitly shown.

Ridge formation is a mode of spontaneous condensation of energy into a small subset of the available volume. As such it resembles the spontaneous organization of dislocations into grain boundaries in a strained crystal or the formation of Prandtl boundary layers in laminar fluid flow [5.6].

To understand the necessary conditions for this condensation, and its consequences, it is useful to consider the general problem of an $M$-dimensional elastic manifold crushed by a hypersphere in $N$-dimensional space. In Ref. [7] we derived the elastic energy functional of the manifold by considering the small thickness limit of an $N$-dimensional elastic solid with an extent $O(L)$ in $M$ directions and a thickness $h$ in $N-M$ transverse directions. Using this formalism we found that a deformed hypersurface $(M=N-1)$ under a specific boundary condition exhibits a ridge with scaling properties analogous to those of Ref. 响. In this Letter we explore the more general situation of an $M$-sheet with a free boundary confined by a small sphere. Using analytic and numerical methods we demonstrate two complementary behaviors:

Case $1(N \geq 2 M)$ The energy $E_{c}$ associated with curvature is distributed uniformly in the manifold and the energy $E_{s}$ associated with strain is negligible by comparison.

Case $2(N=M+1>2)$ The strain energy and curvature energy are comparable, with $E_{s} / E_{b} \simeq 0.2$. Both energies are condensed into a network of narrow, $M-1$

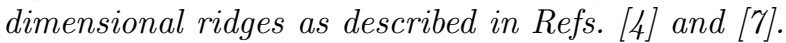

In the remaining cases, namely $M+1<N<2 M$, we anticipate that the strain energy will also be comparable to the curvature energy [8]. We have not investigated these cases in detail.

The differential geometry of the deformed manifold is conveniently discussed using a Euclidean coordinate patch on the flat manifold $\left(x_{\alpha} \mid \alpha \in[1, M]\right)$, called the manifold coordinate system [9]. Any deformation may then be written as a map $\vec{r}(x)$ from the manifold coordinates to $N$-dimensional Euclidian space. The strain tensor is $u_{\alpha \beta}(x)=(1 / 2)\left(\partial_{\alpha} \vec{r} \cdot \partial_{\beta} \vec{r}-\delta_{\alpha \beta}\right)$ and the extrinsic curvature tensor is $\vec{K}_{\alpha \beta}=\partial_{\alpha} \partial_{\beta} \vec{r}[9]$. Under the usual assumptions of linear elasticity theory $\left(u_{\alpha \beta} \ll 1\right.$ and $\left.\partial_{\gamma} u_{\alpha \beta} \ll\left|\vec{K}_{\mu \nu}\right| \ll 1 / h[10]\right)$ the elastic energy functional becomes

$$
\begin{aligned}
E=\int d x^{M} & \left\{\mu\left(\left(u_{\alpha \beta}\right)^{2}+c_{0}\left(u_{\alpha \alpha}\right)^{2}\right)\right. \\
& \left.+\kappa\left(\vec{K}_{\alpha \beta} \cdot \vec{K}_{\alpha \beta}+c_{0} \vec{K}_{\alpha \alpha} \cdot \vec{K}_{\beta \beta}\right)\right\}
\end{aligned}
$$

where $\mu \simeq Y h^{N-M}$ is an effective Lamé coefficient, $\kappa \simeq Y h^{N-M+2}$ is an effective bending rigidity, and $c_{0}$ is a dimensionless constant [7, 11]. Summation over repeated indices is implied. We shall refer to the term quadratic in the strains as the strain energy $E_{s}$, and the term quadratic in the curvatures as the curvature energy 
$E_{c}$. We consider only phantom (not self-avoiding) manifolds in this paper, since we don't expect self-avoidance to alter the qualitative conclusions. Our compressive boundary condition is a frictionless, hard-walled sphere with an initial radius $R_{i}>L$. To crush the manifold the radius is slowly decreased to a final value $R \ll L$.

In our simulations we model a thin elastic 2 -sheet $(M=2)$ using a triangular network of nodes connected by springs (see Fig. 1), following the work of Seung and Nelson [12]. The discretized strain energy is the sum over the Hooke's law energy of each spring

$$
E_{s}=\sum_{i} \frac{1}{2} c_{s}\left(l_{i}-l_{0}\right)^{2}
$$

where $c_{s}$ is a spring constant controlling the strain energy $l_{i}$ is the length of spring $i$, and $l_{0}$ is the lattice constant.

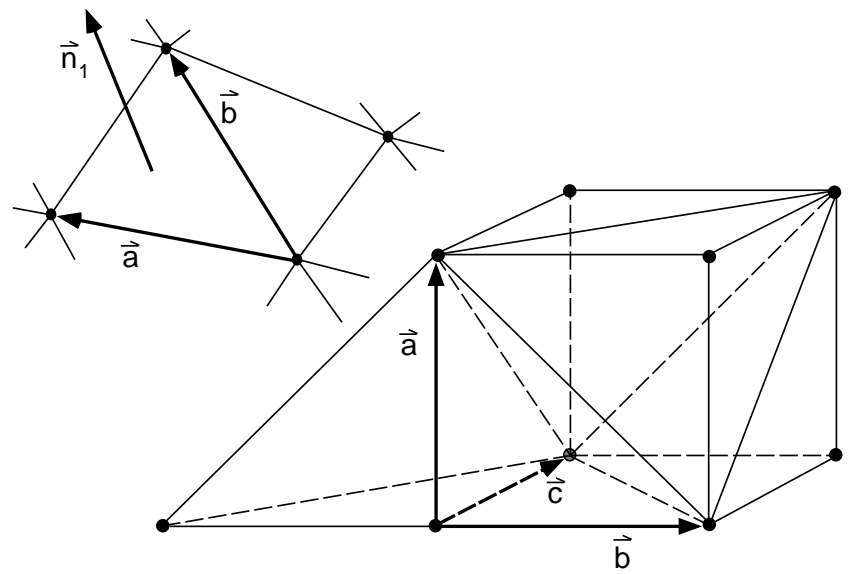

FIG. 1. A portion of the triangular lattice and the simple cubic lattice used to discretize a 2 -sheet $(M=2)$ and a 3 -sheet $(M=3)$ respectively.

To discretize the curvature energy it is convenient to begin by defining the normal tensor for a two-dimensional lattice in $N$-dimensional Euclidean space. If $\vec{a}(x)$ and $\vec{b}(x)$ are two independent vectors tangent to the 2 -sheet at $x$, then we may define a normal tensor via

$$
(\mathrm{n}[a, b])_{i_{1} \cdots i_{N-2}}=\epsilon_{i_{1} \cdots i_{N}} a_{i_{N-1}} b_{i_{N}}
$$

where $a_{i}$ and $b_{i}$ are the Cartesian components of $\vec{a}$ and $\vec{b}$ and $\epsilon$ is the antisymmetric Levi-Civita tensor. In 3space this reduces to the usual normal vector $\mathrm{n}=\vec{a} \times \vec{b}$. Defining the inner product

$$
\mathrm{n}_{1} \cdot \mathrm{n}_{2}=\frac{1}{(N-M) !}\left(\mathrm{n}_{1}\right)_{i_{1} \cdots i_{N-M}}\left(\mathrm{n}_{2}\right)_{i_{1} \cdots i_{N-M}}
$$

with $M=2$, the unit normal tensor may be expressed $\hat{\mathrm{n}}=\mathrm{n} / \sqrt{\mathrm{n} \cdot \mathrm{n}}$. By substituting $\vec{a}=\partial_{1} \vec{r}$ and $\vec{b}=\partial_{2} \vec{r}$, it is straightforward to prove that $\partial_{\alpha} \mathrm{n} \cdot \partial_{\alpha} \mathrm{n}=\vec{K}_{\alpha \beta} \cdot \vec{K}_{\alpha \beta}$. The curvature energy can therefore be represented as the square of the discrete derivative

$$
E_{c}=\sum_{\langle i j\rangle} \frac{1}{2} c_{b}\left|\hat{\mathrm{n}}_{i}-\hat{\mathrm{n}}_{j}\right|^{2}
$$

where $c_{b}$ is a bending constant, $\hat{\mathrm{n}}_{i}$ is the unit normal of triangle $i$ (see FIG. 1), and the sum is taken over each pair of triangles which share a common edge. Comparison with Eq. (1) gives $\mu \simeq c_{s} / l_{0}^{2}, \kappa \simeq c_{b} / l_{0}^{2}$, and $c_{0}=0$ [12]. The effective thickness of the 2 -sheet is determined by the ratio $c_{b} / c_{s} \simeq h^{2}$. We take $\left(c_{b} / c_{s}\right)^{1 / 2} \gtrsim l_{0} / 6$ to minimize lattice effects in our simulations.

The discrete model of an elastic 3 -sheet $(M=3)$ is qualitatively similar. The 3 -sheet is a simple cubic lattice of nodes connected by springs. As shown in Fig. 1 , there are edge springs with constant $c_{e}$ and length $l_{0}$ and diagonal springs with constant $c_{d}$ and length $\sqrt{2} l_{0}$. The requirement that the lattice be elastically isotropic for small strains is $c_{d}=2 c_{e}$. To calculate the curvature energy we first define the normal tensor on a 3-manifold in $N$-space

$$
(\mathrm{n}[a, b, c])_{i_{1} \cdots i_{N-3}}=\epsilon_{i_{1} \cdots i_{N}} a_{i_{N-2}} b_{i_{N-1}} c_{i_{N}}
$$

where $\vec{a}(x), \vec{b}(x)$, and $\vec{c}(x)$ span the tangent space of the manifold at $(x)$. Using the inner product Eq. (雨) with $M=3$, we again have $\partial_{\alpha} \mathrm{n} \cdot \partial_{\alpha} \mathrm{n}=\vec{K}_{\alpha \beta} \cdot \vec{K}_{\alpha \beta}$. Each unit cube is conceived as five adjacent tetrahedra with the springs as edges. Each cube then has four "corner" tetrahedra surrounding one "middle" tetrahedron. The sum in Eq. (5) is taken over all pairs of tetrahedra that share a common triangular face (see Fig. 1). Lastly, we relate the bending constant $c_{c c}$ for a pair of corner tetrahedra to the bending constant $c_{c m}$ for a corner-middle pair. Isotropy requires $c_{c m}=2 c_{c c}$.

The initial condition in our simulations is a hexagonal 2 -sheet $(M=2)$ or a roughly spherical 3 -sheet $(M=3)$ with a small random displacement added to the positions of the nodes [13]. We model a hyperspherical container of radius $r_{0}$ with the potential $V_{\text {sphere }}=\sum_{i}\left(r_{i} / r_{0}\right)^{12}$, where $r_{i}$ is the distance from the origin to node $i$. We use the rms radius of the manifold as our measure of the confining radius $R$ : $R^{2}=\sum_{i} r_{i}^{2} /\left(\sum_{i} 1\right)$. To crush the manifold, the value of $r_{0}$ is repeatedly decreased, and the energy of the manifold minimized at each step using a conjugate gradient routine [14]. Using this method a hexagonal 2-sheet with long diameter $L=160 l_{0}$ may be crushed to a radius $r_{0}=20 l_{0}$ in three days of CPU time on an IBM RISC 6000. An approximately spherical ball with diameter $L=30 l_{0}$ may be crushed to $r_{0}=6.0 l_{0}$ in about five days.

The simplest instance of Case 1 is a thin elastic rod crushed within a circle in 2-space. As the radius of the circle is decreased the rod buckles and develops a curvature of $O(1 / R)$. Because the rod is free to reptate parallel to its length, its strain energy remains identically zero. Analogously, a thin plate in 4space can curl without strain into an arbitrarily small 3 -sphere. One possible embedding which demonstrates 
this is $\vec{r}=\rho\left[\cos \left(x_{1} / \rho\right), \sin \left(x_{1} / \rho\right), \cos \left(x_{2} / \rho\right), \sin \left(x_{2} / \rho\right)\right]$ where $\rho=R / \sqrt{2}$. Note that $r^{2}=R^{2}$ everywhere. One can verify by substitution that the strain tensor $u_{\alpha \beta}$ is identically zero, $\left|\vec{K}_{11}(x)\right|=\left|\vec{K}_{22}(x)\right|=\sqrt{2} / R$, and $E_{t o t} \simeq \kappa(L / R)^{2}$. The key feature of this embedding is the separation of the manifold coordinates into independent, 2-dimensional subspaces of 4-space. Analogously, whenever $N \geq 2 M$ we may write the embedding

$$
\begin{gathered}
\vec{r}=\rho\left[\cos \left(x_{1} / \rho\right), \sin \left(x_{1} / \rho\right), \cos \left(x_{2} / \rho\right), \sin \left(x_{2} / \rho\right),\right. \\
\left.\ldots, \cos \left(x_{M} / \rho\right), \sin \left(x_{M} / \rho\right), 0,0, \ldots\right]
\end{gathered}
$$

where $\rho=R / \sqrt{M}$. This embedding is an existence proof that the manifold need not strain during the compression. It is also the global minimum of the elastic energy at fixed $R$ (neglecting corrections within $R$ of the manifold boundary). This deformation removes the isotropy of the manifold whenever $M>1$. Thus, $e . g$. in order to return to the starting position $\vec{r}$ one must move $\sqrt{2}$ as far in the $(1,1)$ direction as in the $(1,0)$ direction. With this broken symmetry comes a degeneracy. For every distinct orientation of the coordinate system $\left\{x_{1}, \ldots, x_{M}\right\}$, Eq. (7) yields a new minimum-energy configuration.

Our simulations of a 2-sheet in 4-space and a 3-sheet in 6-space behave as anticipated for Case 1. In both instances we observe the minimum energy embedding discussed above, with a curvature energy density uniform to $10 \%$ and negligible strain energy $E_{s} \lesssim 10^{-3} E_{c}$. The only significant deviation from the ideal embedding is that the manifold flattens out within $R$ of the manifold edge. As a result, the curvature energy density decays to zero near the edge and there is a systematic downward correction to the total energy of the form $E_{t o t} \sim(L-\text { (const.) } R)^{M} / R^{2}$. Depending upon the initial condition, we also observe several metastable states with a nonuniform curvature energy density and a higher total energy. As compression proceeds these metastable states make the transition to the minimum energy embedding.

Our understanding of Case 2 deformations is incomplete. It is based on previous analytic and numerical work for a variety of simple deformations, including some on general hypersurfaces [4, []. These cases all show the condensation of the elastic energy into a fraction $O(h / X)^{1 / 3}$ of the manifold volume, suggesting that a generic compression yields similar condensation. The simple cases also exhibit a strict proportionality between strain and curvature energy: $\lim _{(h / X) \rightarrow 0} E_{s}=E_{c} / 5$.

We simulated Case 2 deformations using a 2 -sheet in 3 -space and a 3 -sheet in 4 -space. For the 2 -sheet we see the anticipated spontaneous formation of a network of linear ridges. Fig. 2 shows the curvature energy density in the manifold coordinates of a hexagonal 2-sheet with long diameter $L=160 l_{0}$ crushed to a radius $R=27 l_{0}$. About $40 \%$ of the total curvature energy occupies just $2 \%$ of the total area. This is due to the formation of point vertices in the 2 -sheet. These vertices are the tips of cone-like deformations. The next $40 \%$ occupies $20 \%$ of the area. We see that this energy is condensed into a network of narrow ridges which connect the vertices. The strain energy density is similarly localized. The ratio $E_{s} / E_{c} \approx 10 \%$. This is consistent with the deviations from the asymptotic value $E_{s} / E_{c}=1 / 5$ reported by Lobkovsky for short ridges $X \approx 10 h$ 顿.

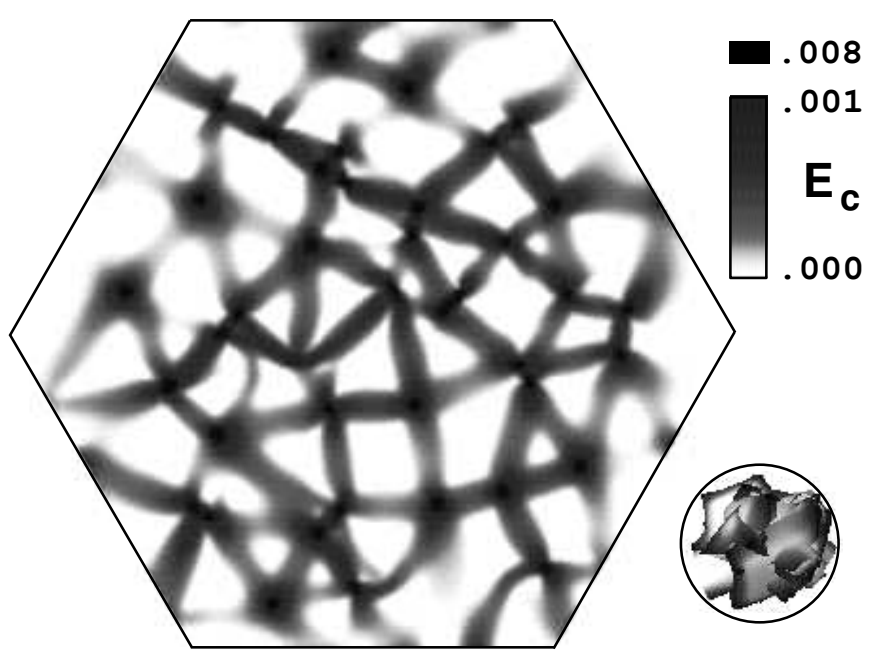

FIG. 2. Curvature energy distribution in a hexagonal 2-sheet with moduli $c_{s}=1.0$ and $c_{b}=.025$ and long diameter $L=160 l_{0}$ crushed to a radius $r_{0}=27 l_{0}$. Darker regions have higher energy density. The crumpled configuration is shown in the lower right.

Fig. 2 suggests that the ridges forming the network are of comparable length. This length is similar to the diameter of the confining sphere shown in the lower right corner. If we make the scaling hypothesis that the mean ridge length $\bar{X} \simeq R$, then the number of ridges in the 2 -sheet scales like $(L / R)^{2}$. Since the energy of a single ridge scales as $Y h^{3}(R / h)^{1 / 3}[\mathbb{1}$, the total elastic energy $E_{\text {tot }}$ should obey $E_{t o t} \simeq Y h L^{2}(h / R)^{5 / 3}$. Our simulation results are consistent with $E_{t o t} \sim R^{-5 / 3}$ 15]. However, due to boundary effects and the small values of the aspect ratio $L / h \lesssim 300$ the evidence for this scaling is only suggestive.

Our simulations of an elastic 3 -sheet in 4 -space attained aspect ratios of only $L / h \simeq 30$. Since the ridge properties derived in Ref. [7] are well-defined only in the limit that the ridge is much longer than $h$, these simulations provide only qualitative support for Case 2 . The simulations show the anticipated concentration of strains and curvatures into linear and planar structures. Fig. 3 shows two curvature energy isosurfaces in the manifold coordinates of a 3 -sheet with diameter $L=30 l_{0}$ crushed to $r_{0}=12 l_{0}$. The upper isosurface encloses $65 \%$ of the total curvature energy and $16 \%$ of the volume. We see distinct planar structures. The lower isosurface encloses $23 \%$ of the curvature energy and only $3 \%$ of the volume. The energy is concentrated on the set of lines where planes intersect. The lines are the analogs of the point-like vertices in FIG. 2. The ratio $E_{s} / E_{c}$ is $15 \%$.

We anticipate scaling behavior for the energy of a 
crushed hypersurface analogous to that for a crushed 2sheet. If the linear size of the ridges is roughly the diameter of the confining sphere, we may generalize the argument for the 2-sheet to infer $E_{t o t} \simeq Y h L^{M}(h / R)^{5 / 3}$ [7]. The limitations of our simulation prevented us from testing this prediction.

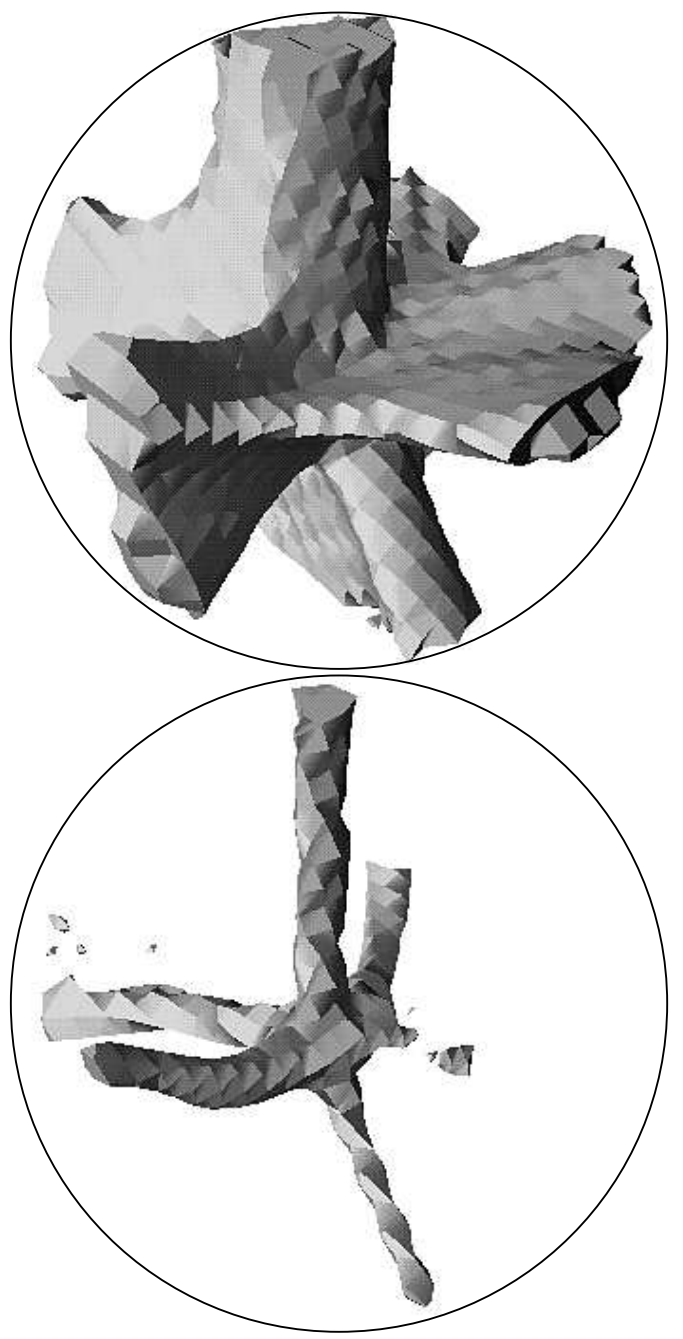

FIG. 3. Curvature energy isosurfaces in the manifold coordinates of an elastic 3 -sheet $\left(L=30 l_{0}\right)$ in 4 -space crushed to a radius $r_{0}=13 l_{0}$. The top (bottom) surface encloses $65 \%(23 \%)$ of the total energy in $16 \%(3 \%)$ of the volume.

This work has implications for real crumpled sheets. It is the first exploration of the distribution of energy in these sheets and it offers qualitative support for the model of a crumpled sheet as a network of ridges [4]. The possibility of crushing without strain in high dimensions may be relevant for studies of thermal crumpling in general dimensions 16. More broadly, this work identifies a new mechanism of energy condensation into a small subspace of an available volume. This condensation happens in arbitrary spatial dimensions in one of the simplest or- ganizations of matter - an elastic manifold. Increasingly, the behavior of simple manifolds in general spatial dimensions is invoked to account for fundamental processes [17]. The symmetry-breaking and ridge-forming mechanisms explored here may prove relevant for understanding such behavior.

\section{ACKNOWLEDGMENTS}

The authors thank Robert Geroch, Alex Lobkovsky, and Jeff Harvey for helpful discussions. This work was supported in part by the NSF through Grants No. DMR9400379 and DMR-9528957.

$\dagger \quad$ e-mail address: kramer@rainbow.uchicago.edu

[1] The Collected Works of Theodore von Karman (Butterworths Scientific, London, 1956) vol. IV, p. 7.

[2] F. Plouraboue and S. Roux, Physica A 227, 173 (1996).

[3] T. Witten and H. Li, Europhys. Lett. 23, 51 (1993).

[4] A. Lobkovsky et al., Science 270, 1482 (1995); A. Lobkovsky, Phys. Rev. E 53, 3750 (1996); A. Lobkovsky and T. Witten. Submitted to Phys. Rev. E.

[5] D. Hull, Introduction to Dislocations (Pergammon Press, New York, 1975).

[6] A. Walz, Boundary Layers of Flow and Temperature (MIT Press, Cambridge, 1969).

[7] E. Kramer, cond-mat/9608050. Submitted to J. Math. Phys.

[8] The proof of this conjecture for $N=M+1$ and $N=$ $M+2$ will be presented in a future paper.

[9] L. Eisenhart, Differential Geometry (Ginn and Company, New York, 1909); R. Millman and G. Parker, Elements of Differential Geometry (Prentice-Hall, New Jersey, 1977).

[10] L. Landau and E. Lifshitz, Theory of Elasticity (Pergamon Press, New York, 1959).

[11] The Young's modulus has units of energy per unit $N$ volume, so $\mu$ has units of energy per unit $M$-volume.

[12] H. Seung and D. Nelson, Phys. Rev. Lett. 57, 791 (1986).

[13] The final configuration of a crumpled hypersurface $(N=$ $M+1)$ depends strongly on the random initial condition, but the qualitative appearance of the ridge network persists and the final energy varies by only $10 \%$.

[14] W. Press et al., Numerical Recipes (Cambridge University Press, New York, 1989).

[15] The simulation results are also consistent with $E_{t o t} \sim$ $R^{-2}$, the dependence expected for Case 1.

[16] Y. Kantor, M. Kardar and D. R. Nelson, Phys. Rev Lett 57791 (1986); Statistical Mechanics of Membranes and Surfaces, D. Nelson, T. Piran, and S. Weinberg, eds. (World Scientific, Singapore, 1989).

[17] See e. g. E. Witten, Nucl. Phys. B 443 (1995). 